\title{
Doğal Kabak Çekirdeği Kabuğunun Bor Adsorpsiyon Kapasitesi
}

\author{
Hakan ÇELEBIं* \\ Aksaray Üniversitesi, Mühendislik Fakültesi, Çevre Mühendisliği Bölümü, Aksaray \\ (ORCID: 0000-0002-7726-128X)
}

\begin{abstract}
Öz
Endüstriyel gelişmelere bağlı olarak birçok sanayide ve yüzey sularında kullanılan bor bileșiklerinin konsantrasyonu artmaktadır. Bu nedenle bor giderimi hem insan sağlığı hem de sağlıklı tarım ürünü yetiştirilebilmesi açısından oldukça önemlidir. Bu çalışmada, doğal kabak çekirdeği kabuğunun adsorpsiyon kapasitesi araştırılmıştır. Çalışma için, çözeltinin $\mathrm{pH}$ 'sının, kabak çekirdeği kabuğu konsantrasyonlarının ve temas süresinin adsorpsiyon sürecine etkisi sabit bir karıștırma hızı $(150 \mathrm{rpm})$ ve sıcaklığı $\left(20{ }^{\circ} \mathrm{C}\right)$ altında değerlendirilmiştir. Ayrıca, kabak çekirdeği kabuğu, taramalı elektron mikroskobu, X ışını floresansı ve Fourier dönüşümü kızılötesi teknikleri kullanılarak tanımlanmıştır. Elde edilen deneysel veriler kesikli proseste farklı izoterm ve kinetik modelleri kullanılarak analiz edilmiştir. Adsorpsiyon işlemi için optimum koşullar adsorbent dozu $=2 \mathrm{~g} / \mathrm{L}, \mathrm{pH}=5.13$ ve temas süresi $=5$ dakika olarak belirlenmiştir. Kabak çekirdeği kabuğunun maksimum bor arıtma verimi, ideal şartlarda yaklaşı $\% 88$ olarak elde edilmiştir. Langmuir izotermine dayalı maksimum adsorpsiyon kapasitesi $12.61 \mathrm{mg} / \mathrm{g}$ şeklinde olup bor elementinin kabak çekirdeği kabuğu üzerine adsorpsiyonu yalancı ikinci derece kinetik ile tanımlanmıştır. Sonuç olarak, bor elementinin, kabak çekirdeği kabuğu kullanılarak sulu çözeltiden etkili bir şekilde çıkarılabileceği kaydedilmiştir.
\end{abstract}

Anahtar kelimeler: Adsorpsiyon, bor, kabak çekirdeği kabuğu.

\section{Boron Adsorption Capacity of Natural Pumpkin Seed Shell}

\begin{abstract}
Due to industrial developments, the concentration of boron compounds used in many industries and surface waters increases. Therefore, boron removal is very important in terms of both human health and healthy agricultural product. In this study, the adsorption capacity of natural pumpkin seed shell was investigated. For the study, the effect of $\mathrm{pH}$ of the solution, pumpkin seed shell concentrations and contact time on the adsorption process was evaluated under a constant mixing speed $(150 \mathrm{rpm})$ and temperature $\left(20{ }^{\circ} \mathrm{C}\right)$. In addition, pumpkin seed shell, scanning electron microscopy, X-ray fluorescence and Fourier transform were identified using infrared techniques. The obtained experimental data were analyzed by using different isotherm and kinetic models in the batch process. Optimum conditions for adsorption were determined as adsorbent dose $=2 \mathrm{~g} / \mathrm{L}, \mathrm{pH}=5.13$ and contact time $=5$ minutes. The maximum boron treatment efficiency of the pumpkin seed shell was obtained as approximately $88 \%$ under ideal conditions. The maximum adsorption capacity based on Langmuir isotherm is $12.61 \mathrm{mg} / \mathrm{g}$ and the adsorption of boron on pumpkin seed shell is defined by pseudo-second-order kinetic. As a result, it was noted that the boron element can be effectively removed from the aqueous solution using pumpkin seed shell.
\end{abstract}

Keywords: Adsorption, boron, pumpkin seed shell.

\section{Giriș}

Bor, dünyadaki hidrosfer ve litosfer bölgelerinde geniş çapta dağılmış bir mikro elementtir. Doğada, bor her zaman başka elementlere sahip bileşikler olarak bulunmaktadır. Bor, bileşiklerinin çevreye önemli miktarlarda yayılmasına neden olan çok sayıda endüstriyel uygulamalar vardır [1,2]. Bor ve bileşikleri, cam, elektronik, seramik, porselen, yarı iletkenler, kozmetik ve kişisel bakım ürünleri, halılar ve yanmaz kumaşlar, tarımsal ilaçlar, katalizör ve temizlik ürünleri, eczacılık ürünleri, sabunlar ve deterjanlar, gübreler gibi birçok endüstride yaygın olarak kullanılmaktadır (Şekil 1) [3,4]. Bor içeren ürünlerin

*Sorumlu yazar: hakanaz.celebi@gmail.com

Geliş Tarihi: 30.12.2019, Kabul Tarihi: 27.09.2020 
yaygın kullanımı nedeniyle, bor atıkları günümüzde ciddi bir problem haline gelmiştir [5,6]. Ayrıca, bor elementi bitki, hayvan ve insan büyümesinde de önemli bir rol oynamaktadır. Ancak, hem eksikliği hem de fazlalılığ günlük alımlarda toksik etkilere neden olabilmektedir. Uzun süreli bor konsantrasyonu yüksek suların ve besinlerin kullanımı sonucunda nörolojik ve zihinsel sorunlar, kalp-damar- karaciğerböbrek hastalıkları, üreme bozuklukları ve sindirim sistemi gibi çeşitli sağlık sorunları ortaya çıkabilmektedir [7]. Bununla birlikte, aşırı bor seviyeleri bitki ve meyve-sebze gelişimi açısından risk oluşturmaktadır. Özellikle, yapraklar ve meyvelerde sarı lekeler gözlenmekte ve ölümler gerçekleşmektedir. Bu durum da tarımsal açıdan mahsul verimini önemli ölçüde azaltmaktadır.

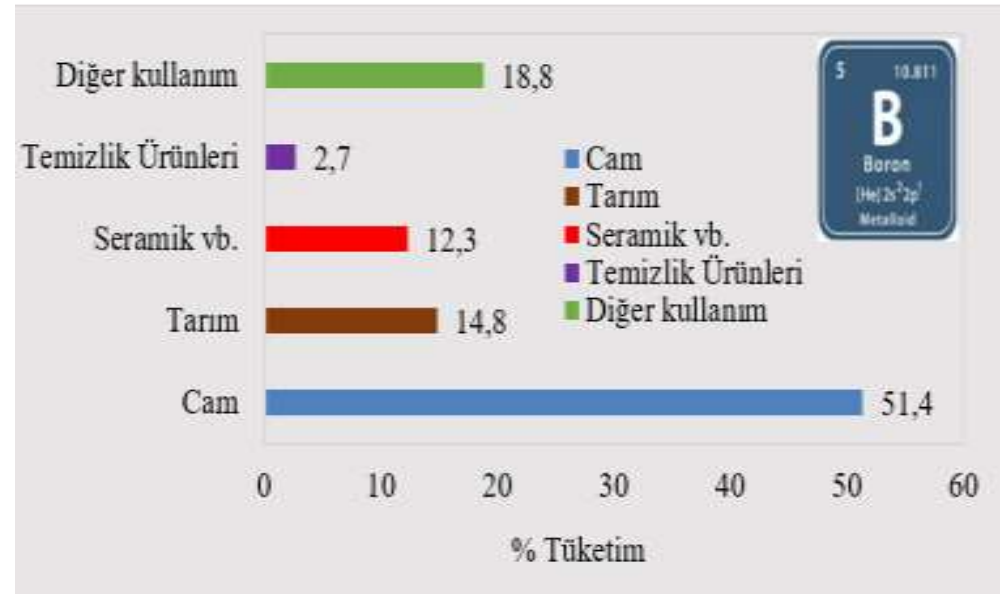

Şekil 1. Sektör bazında bor tüketim dağılımı

Dünyadaki bor rezervlerinin \%72'sinden fazlası Türkiye'de bulunmaktadır [8]. Bu rezervlerin işletilmesi sırasında oluşan sıvı ve katı atıklar, ekolojik ortamların kirlenmesine neden olmaktadır. Türkiye' de sulamada kullanılan temiz suyun kirlenmesine neden olan toksik elementlerin başında bor gelmektedir. Ayrıca, sulama için geri kazanılabilen endüstri atıksuları da kullanılmaktadır. Sulama amaçlı kullanılan endüstri atıksularında bor derişimi limit değerlerin üzerine çıktığı zaman, bitkilere veya suda yaşayan canlılara zarar verebilmektedir. Ayrıca, sulama suyunda ve toprakta bulunan bor miktarı, ürünlerin yetiştirilmesinde ve kalitesinde önemli bir rol oynar $[4,9,10]$. Sonuç olarak, bazı ülkelerde, önerilen bor konsantrasyonu sulama suyu aralığ 10,3 ile $1 \mathrm{ppm}$ arasındadır. İçme suyunda borun konsantrasyonu, Dünya Sağlik Örgütü tarafindan $\leq 2.4 \mathrm{mg} / \mathrm{L}$ ve Avrupa Birliği tarafindan $\leq 1.0$ $\mathrm{mg} / \mathrm{L}$ olarak belirlenmiştir. Türkiye' deki Su Kirliliği Kontrolü Yönetmeliğí'nde, içilecek su olarak öngörülen kullanılabilecek 1. kalite suda borun olması gereken maksimum miktarı $1.0 \mathrm{mg} / \mathrm{L}$ 'dir $[11,12]$. Ayrıca sulama suyu için ise bor değeri $0.5 \mathrm{mg} / \mathrm{L}$ olarak belirlenmiştir. Bu nedenle içerisinde bor bulunan atıksuların, doğaya salınabilmesi için, belirlenen değere getirilmesi gereklidir. Özellikle atıksuların yeninden kullanımı ve geri kazanımı kapsamında sulamada sorun oluşturabilecek bor vb. maddelerin arıtılması önem kazanmaktadır.

Sulu çözeltilerden borun giderilmesi için farklı metotlar bulunmaktadır. Bunlar oldukça geniş uygulama alanı olan kimyasal çökeltme, iyon değişimi, adsorpsiyon, elektrokoagülasyon, çözücü ekstraksiyonu ve membran teknolojileri, ters ozmoz, ileri ozmoz, nanofiltrasyon, ultrafiltrasyon ve fitodüzenlemeden oluşmaktadır [13-16]. Bununla birlikte, adsorpsiyon dışında bu teknolojilerin pahalı kimyasallara duyduğu gereksinimleri, düşük giderme verimleri ve arıtma işleminde ikincil atıkların çıkması gibi birçok dezavantajları bulunmaktadır. Borun giderilmesi için bildirilen yöntemler arasında adsorpsiyon, yüksek adsorpsiyon kapasitesi, kolay işlenebilirlik, etkili bor alımı, kolay rejenerasyon, uygun hazırlık, dikkat çekici geri dönüşüm performansı ve nispeten düşük maliyet ve düşük borlu sulu ortamlarda kullanılabilirliği nedeniyle en çok araştırılan işlemlerdendir [17,18]. Sulu çözeltiden borun çıkarılması için birçok adsorbent (Fe (III) modifiye bentonit kil, seçici reçineler, lifler, aktif karbon, oksitler ve hidroksitleri, tabakalı çift hidroksitleri, fonksiyonel polimerler, doğal malzemeler, farklı kil grupları vb.) kullanılmıştır [19-25].

Günümüzde etkili atıksu yönetimi için su kaynaklarının çevre dostu, uygun maliyetli ve daha az karmaşı olarak arıtılması gerekmektedir. Kirleticilerin atıksudan uzaklaştııılmasında kullanılan çeşitli yöntemler arasında adsorpsiyonun üstün olduğu yapılan çalışmalarla bulunmuştur. Araştırmacılar daha 
önce, bor adsorpsiyonundaki gelişimin, adsorbentlerin türüne bağlı olduğunu ortaya koymuşlardır. Düşük bor konsantrasyonlarında, adsorpsiyon, bor giderme yöntemleri arasında en faydalı ve ekonomik tekniktir. Literatürde çok çeşitli adsorbent malzeme olmasına rağmen atık olarak nitelendirilen ve çöplere atılan materyallerin kullanımı hem arıtım hem de atığın atıkla giderilmesi açısından yenilikçi bir yaklaşımdır. Özellikle bor için deneysel çalışmalarda modifiye edilmiş malzemelerin tercih edilmesinden dolayı bu çalışmada hiçbir işleme uğramamış tarımsal nitelikli atıkları tercih etmek bir avantaj olarak görülmektedir. Bu çalışma için adsorpsiyonda kabak çekirdeği kabukları (KÇK) genellikle bollukları, düşük maliyetli, kolay erişilebilir ve uygulanabilir olmaları nedeniyle doğal bir adsorbent olarak kullanılmıştır. Bu çalışmada, sulu çözeltideki bor iyonlarının adsorpsiyon yoluyla giderilmesi ve adsorpsiyona etki eden faktörler incelenmiştir. Bu nedenle, optimum çözelti pH'sının, temas süresinin ve adsorbent miktarının adsorplama kapasitesine olan etkileri araştırılmış ve elde edilen sonuçların değerlendirilmesi yapılmıştır.

\section{Materyal ve Metot}

\subsection{Kullanılan Kimyasallar ve Stok Çözelti}

Deneylerde kullanılan tüm kimyasal maddeler analitik saflık derecesinde olup $1000 \mathrm{mg} / \mathrm{L}$ 'lik bor stok çözeltisi hazırlamak için $5.730 \mathrm{~g} \mathrm{H}_{3} \mathrm{BO}_{3}$ tartılarak 1 L'lik balon jojede deiyonize su (kimyasal direnci: $18 \mathrm{M} \Omega \mathrm{cm} ; 1.2 \mu \mathrm{g} / \mathrm{L}$ toplam organik karbon) ile çözüldükten sonra stok çözeltiden seyreltilerek hazırlanan çözeltiler kullanılmıştır. Bu çalışmada kullanılan kimyasallar, çözeltilerin $\mathrm{pH}$ ayarlamaları için $0.1 \mathrm{M} \mathrm{HCl}$ ve $\mathrm{NaOH}$ kimyasalları Merck (Merck KGaA, Darmstadt, Germany) firmasından temin edilmiştir. Tüm cam aletler kullanımdan önce birkaç kez ultra saf su ile temizlenmiştir. Adsorpsiyondan önce, çözeltilerin başlangıç pH değerleri dijital pH metre (HANNA pH 211) ile ayarlanmıştır.

\subsection{Adsorbentin Hazırlanışı ve Karakterizasyonu}

Araştırmada kullanılan evsel atık ham maddesi yerel evlerden ve marketlerden toplanmıştır. KÇK laboratuvar ortamında elle çıkarılmış ve adsorbent olarak kullanılmadan önce, herhangi bir toz, kir, renk sorunu oluşmaması için, 24 saat boyunca $100{ }^{\circ} \mathrm{C}$ sıcaklıkta saf suyla birkaç kez yıkama işlemi gerçekleştirilmiştir. Yıkama işleminden sonra $\mathrm{KÇK} 70{ }^{\circ} \mathrm{C}$ etüvde kurutulmuş ve bir kahve öğütücü kullanılarak toz haline getirilmiştir. Deneyden önce, başka herhangi bir kimyasal veya fiziksel işlem kullanılmamıştır. Adsorbenti depolayabilmek için plastik kutular tercih edilmiştir. Adsorbent olarak etkinliği incelenecek olan KÇK'nın fonksiyonel grupları 400-4000 $\mathrm{cm}^{-1}$ aralığında spektrumlarda Fourier Dönüşümlü Kızılötesi Spektrometre (FTIR) (Thermo Scientific-Nicolet iS5) ile belirlenmiştir. KÇK'ların yüzey morfolojisi ve element cinsi ise sırası ile taramalı elektron mikroskobu (TEM) (Hitachi-SU 1510) ve X-Işınları Floresans Spektrometresi (XFS) (Rigaku-NEX-CG) cihazları ile incelenmiştir. Quantachrome-Quadrasorb Evo 4 marka cihaz ile Brunauer, Emmett ve Teller (BET) metoduyla $77 \mathrm{~K}$ 'deki sıvı azot ortamında, azot $\left(\mathrm{N}_{2}\right)$ gazı adsorpsiyonu tekniğine dayalı olarak yüzey alanı ve gözeneklilik ölçümü yapılmıştır.

\subsection{Sulu Çözeltide Bor (B) Adsorpsiyonu}

Adsorpsiyon çalışmaları kesikli düzende çalışan cam ekipmanlarda gerçekleştirilmiştir. $100 \mathrm{~mL}$ çalışma hacmine sahip 250 mL'lik ağzı kapalı erlenler kullanılmıştır. Hazırlanan erlenler ZHICHENG analitik model bir termal çalkalayıcıda 150 rpm'lik sabit karıştırma hızı ve $25{ }^{\circ} \mathrm{C}$ sabit sıcaklıkta işleme tabi tutulmuştur. Borun KÇK üzerine adsorpsiyonun şematik olarak gösterimi Şekil 2'de verilmiştir. Adsorpsiyon deneyi için sırası ile temel mekanizmayı belirleyen $\mathrm{pH}$, zaman, adsorbent dozu parametrelerine göre deneyler uygun koşullarda gerçekleştirilmiştir. Adsorplanan miktarın hesaplanması için süzülen numuneler (ICP-OES, 2100DV, Perkin Elmer, USA) marka endüktif olarak eşleşmiş plazma optik emisyon spektrometresi (ICP-OES) cihazına verilmiş ve sonuçlar kaydedilmiştir (Şekil 2). 


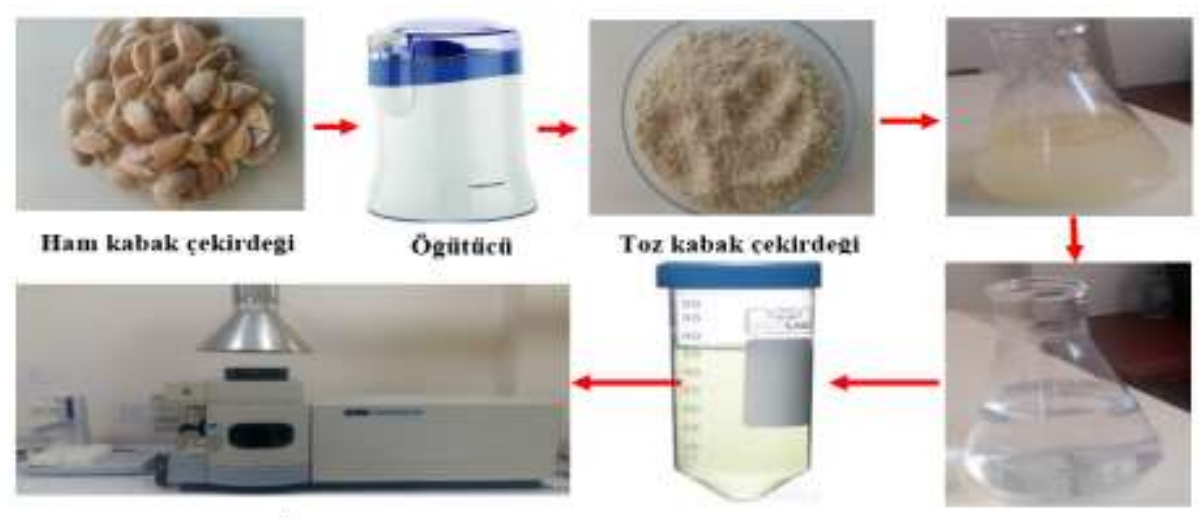

ICP_OES Ölcümū

Şekil 2. Borun KÇK üzerine adsorpsiyonun şematik olarak gösterimi

Adsorpsiyon dengeye ulaştıktan sonra bor adsorpsiyon verimi "AV (\%)" ve adsorbentin birim miktarına bor adsorpsiyon kapasitesi“ " $\mathrm{q}_{\mathrm{e}}(\mathrm{mg} / \mathrm{g})$ ” değerleri aşağıdaki formüllere göre hesaplanmıştır.

$$
\begin{aligned}
& \mathrm{q}_{\mathrm{e}}=\frac{\left(\mathrm{C}_{0}-\mathrm{C}_{\mathrm{e}}\right) \times \mathrm{V}}{1000 \times \mathrm{m}} \\
& \mathrm{AV}(\%)=\frac{\left(\mathrm{C}_{0}-\mathrm{C}_{\mathrm{e}}\right)}{\mathrm{C}_{0}} \times 100
\end{aligned}
$$

Burada; AV: Bor verimi (\%), $\mathrm{C}_{0}$ ve $\mathrm{C}_{\mathrm{e}}$ : Borun ilk ve son konsantrasyonları (mg/L), m: KÇK (mg) miktarı, V: çözeltinin hacmi $(\mathrm{mL})$, qe: KÇK tarafından adsorbe edilmiş deneysel bor miktarı $(\mathrm{mg} / \mathrm{g})$.

\section{Bulgular ve Tartışma}

\subsection{KÇK Adsorbentinin Karakterizasyonu}

KÇK'nin XFS analizine göre elementel bileşimi ve birkaç önemli özellikleri Tablo 1'de gösterilmektedir [26-28]. Çalışmada kullanılan XFS cihazı $\mathrm{C}, \mathrm{H}, \mathrm{O}$ ve $\mathrm{Na}$ gibi elementlerin okumasını gerçekleştiremediğinden diğer cihazlar ile bu elementler değerlendirmeye alınmıştır. Araştırılan KÇK'nın dokusal özellikleri olarak nitelendirilen BET yüzey alanı ve toplam gözenek hacmi sırasıyla $98.38 \mathrm{~m}^{2} / \mathrm{g}$ ve $0.49 \mathrm{~cm}^{3} / \mathrm{g}$ olarak belirlenmiştir. $\mathrm{pH}$ ZPC değeri ise, adsorbentin net bir yüzey nötrlügüne sahip olduğunda pH'ı belirleyen önemli bir özelliği olup çalışmamızda KÇK'nın pH ZPC değerinin 5.96 (yaklaşık 6.0) olduğu tespit edilmiştir.

\begin{tabular}{|c|c|c|c|c|}
\hline \multicolumn{2}{|c|}{ XFS Değerleri } & \multicolumn{3}{|c|}{ Bazı Spesifik Özellikler } \\
\hline Elementler & Ăğırlık (\%) & Parametreler & Birimler & Değerler \\
\hline $\mathrm{K}$ & 2.56 & Nem & $(\%)$ & 8.54 \\
\hline $\mathrm{P}$ & 0.65 & Kül & $(\%)$ & 3.48 \\
\hline $\mathrm{Mg}$ & 0.47 & Ham Lif & $(\%)$ & 15.85 \\
\hline$S$ & 0.45 & Ham Protein & $(\%)$ & 29.15 \\
\hline $\mathrm{Ca}$ & 0.26 & Peroksit & (meq/kg) & 13.68 \\
\hline $\mathrm{Cl}$ & 0.15 & İyot & (gL 100/g) & 82.71 \\
\hline $\mathrm{Zr}$ & 0.09 & Spesifik ağırlık & $\left(\mathrm{g} / \mathrm{cm}^{3}\right)$ & 0.87 \\
\hline $\mathrm{Si}$ & 0.04 & Erime Noktası & $\left({ }^{0} \mathrm{C}\right)$ & 31.89 \\
\hline $\mathrm{Fe}$ & 0.02 & Donma Noktas1 & $\left({ }^{0} \mathrm{C}\right)$ & 12.99 \\
\hline
\end{tabular}

Tablo 1. KÇK'nın elementel bileşimi ve bazı özellikleri

KÇK'nın yüzey kimyası, Şekil 4'teki gibi FT-IR spektroskopisi kullanılarak değerlendirilmiştir. Şekil 3'ten, KÇK'nın 3305.38, 2922.85-2161.04 ve 598.38-1743.85 $\mathrm{cm}^{-1}$ civarında farklı bantlar gösterdiği görülmektedir. Bu bantlar hidroksil ve disülfit gruplarının germe titreşimlerine, $\mathrm{O}-\mathrm{H}, \mathrm{C}-\mathrm{H}$, 
$\mathrm{C}-\mathrm{N}, \mathrm{S}=\mathrm{O}$, karboksil grubu ve $\mathrm{C}-\mathrm{O}-\mathrm{C}$ grubunun simetrik ve asimetrik gerilmelerine işaret etmektedir. Bu sonuçlar daha önceki çalışmalarla benzerlik taşımaktadır [29-32].

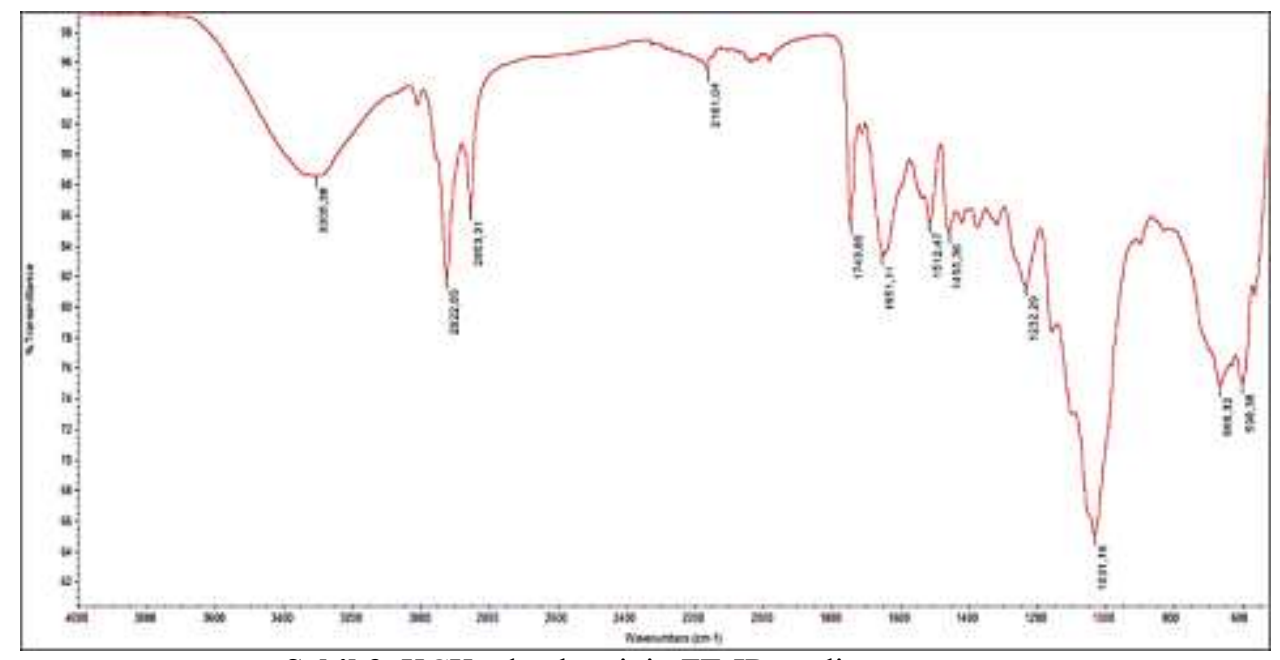

Şekil 3. KÇK adsorbentinin FT-IR analiz görüntüsü

Hazırlanan KÇK'nın taramalı elektron mikrografı ve element analizi Şekil 4'te gösterilmektedir. KÇK birçok sayıda büyük ve orta ölçekli gözeneklere sahiptir. Bu gözenekler sayesinde bor elementini yakalamak ve adsorbe etmek kolaydır. Bor yüklü adsorbent görüntülerine bakıldığında, bor moleküllerinin yüzeyi kapladığı görülmektedir. Bu nedenle, bor yerleşimi doğrultusunda, tek tabakalı adsorpsiyon elde edilebilir. KÇK'daki element değiş̧ikliği analizine göre ağırlıkça; C (\% 78.38), O (\% 21.55), K (\% 0.06) ve S (\% 0.02) elementlerinden oluşmaktadır. Adsorpsiyon sonrası gözenekler bor ile kaplanmış olup bu durum KÇK'nın BET yüzey alanı ve toplam gözenek hacmi ile de desteklenmiştir. XFS ve FT-IR analizlerinde olduğu gibi taramalı elektron mikrografı ve element analizinde de önceki çalışmalar ile KÇK değerleri uygunluk göstermektedir [33-35].

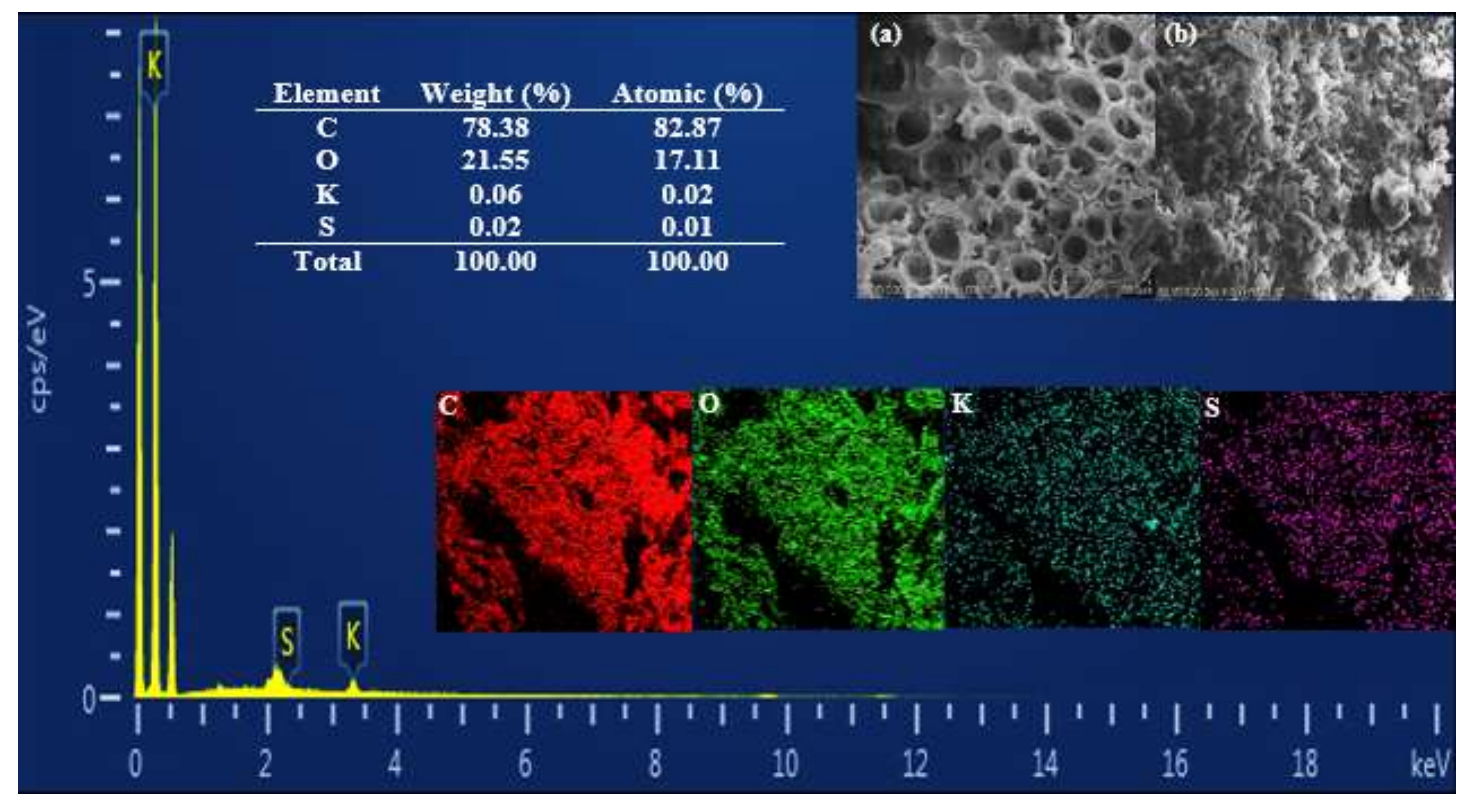

Şekil 4. KÇK'nın taramalı elektron mikrografı ve element analizi (a: adsorpsiyon öncesi, b: adsorpsiyon sonrası, C: karbon, O: oksijen, K: potasyum, S: kükürt)

\section{2. pH'ın Bor Adsorpsiyonu Üzerindeki Etkisi}

pH bir ortam parametresi olup adsorpsiyonu etkileyen en önemli faktördür. $\mathrm{pH}$ hem adsorbenti hem de adsorplanan yapıyı etkileyebilmektedir. $\mathrm{pH}$ değiştikçe ortamda bulunan hidrojen $\left(\mathrm{H}^{+}\right)$, hidroksil $\left(\mathrm{OH}^{-}\right)$ iyonlarının kuvvetli bir şekilde değişmesinden dolayı adsorpsiyon verimi de değişmektedir. Sulu 
sistemlerde inorganik borun baskın formlar1, borik asit $\mathrm{H}_{3} \mathrm{BO}_{3}$ ve oksiborat iyonu $\mathrm{H}_{2} \mathrm{BO}_{3}$ gibi türlerdir. Sulu çözeltilerde borik asit ve borat iyonunun özelliklerini belirleyen en önemli parametre ise ortamın pH'ıdır. Şekil 5a'da görülebileceği gibi, borik asit molekülü düşük ve nötr $\mathrm{pH}$ değerlerinde, borat ise yüksek pH değerlerinde aktif olmaktadır [36]. Şekil 5b'de yaklaşık 2 ila $11 \mathrm{pH}$ aralıklarında KÇK'nın bor adsorpsiyonunu etkilediği görülmüştür. Farklı $\mathrm{pH}$ değerleri arasından yapılan kesikli deneyler sonucunda maksimum bor giderme verimi (\%92) pH 5.13'de bulunmuştur. Asidik düzeye yakın bir ortam için kaydedilen maksimum bor verimi, çözelti içindeki katyonlar ile KÇK'nın adsorpsiyon bölgelerinde fonksiyonel gruplar arasındaki etkileşimin sonucu olabilir. Çalışmamızda kuvvetli alkali koşullar ( $\mathrm{pH}>7.0)$ altında borun çıkarılmasındaki düşüş, tetrahidroksibratın negatif yüklü KÇK yüzeyine elektrostatik olarak bağlanmasından veya borat anyonlarıyla rekabet edebilecek aşırı hidroksil anyonlarının konsantrasyonundan kaynaklanabilir. Ayrıca, adsorbent yüzeyinin yüklenmesine göre adsorpsiyon veriminin şekillendiği bilinen bir gerçektir. Yapılan çalışmalar sonucu, bor adsorpsiyonu için en uygun $\mathrm{pH}$ değerleri 5-10 arası olduğu belirtilmiştir [37-41].

\subsection{KÇK Adsorbentinin Miktarı}

KÇK miktarının borun adsorpsiyon süreci üzerindeki etkisi, $100 \mathrm{~mL}$ bor solüsyonunda $25^{\circ} \mathrm{C}$ 'de 150 rpm'de 0.1-5.0 g aralığında değiştirilerek incelenmiş̧tir. Borun giderim yüzdesi, KÇK dozu arttıkça önce $2.0 \mathrm{~g}$ seviyesine kadar artmış daha sonraki miktarlarda kısmen azalmıştır (Şekil 5d). Bor verim yüzdesi sırasiyla $0.1,0.5,1.0,1.5,2.0,3.0$ ve 5.0 g olan KÇK dozları için \%81.32, 82.19, 82.98, 84.16, 87.15, 80.08 ve 69.86 seviyesine düşmüştür. Bunun etkili adsorbent yüzey alanı ve daha yüksek adsorpsiyon kabiliyeti nedeniyle olduğu söylenebilir. Maksimum bor giderme verimi (\% 87.15), $2.0 \mathrm{~g}$ optimal miktar kullanılarak elde edilmiştir. Birçok araştırmacı, boru atıksudan verimli bir şekilde gidermek için farklı adsorbentler denemiştir. Literatürdeki diğer araştırmalar, çeşitli adsorbentler kullanarak bor adsorpsiyonu için benzer bulgular sunmaktadır. Ancak kullanılan adsorbentlerin çoğu modifikasyona uğramış türden olup ham adsorbent kullanımı oldukça sınırlı seviyededir. KÇK ile elde edilen sonuçlar önceki araştırmaların modifiye adsorbent sonuçlarına benzerlik göstermektedir [42-45].

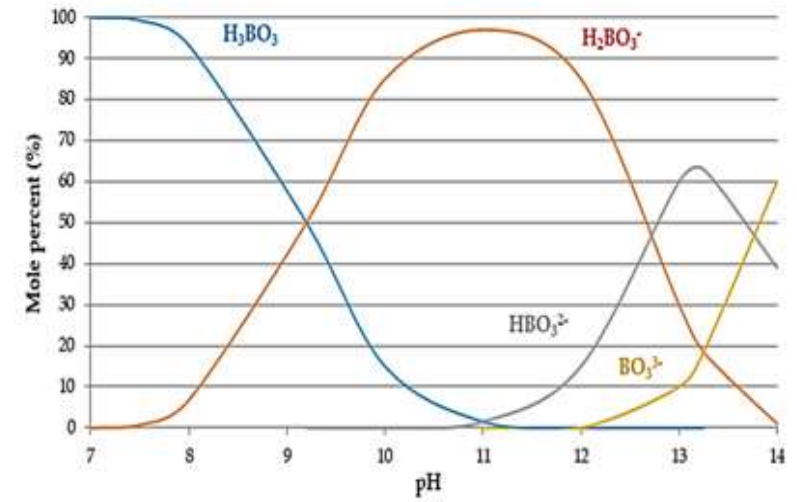

(a) $\mathrm{pH}$ ile bor bileșiklerinin değişimi

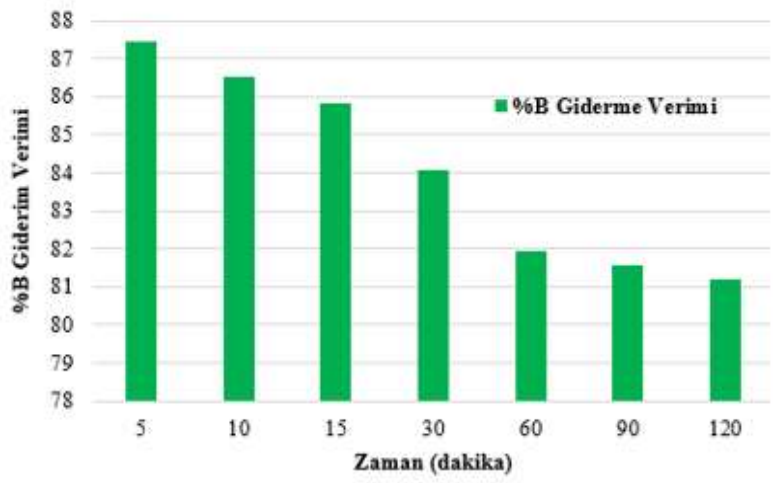

(c) Bor giderimine zamanı etkisi

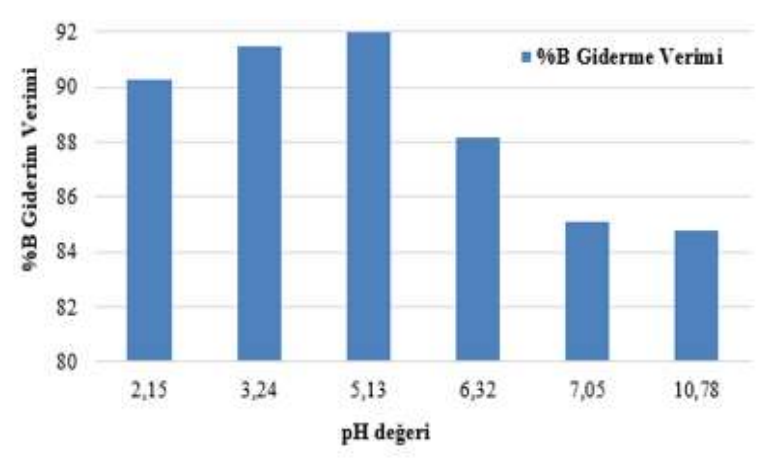

(b) Bor giderimine $\mathrm{pH}$ etkisi

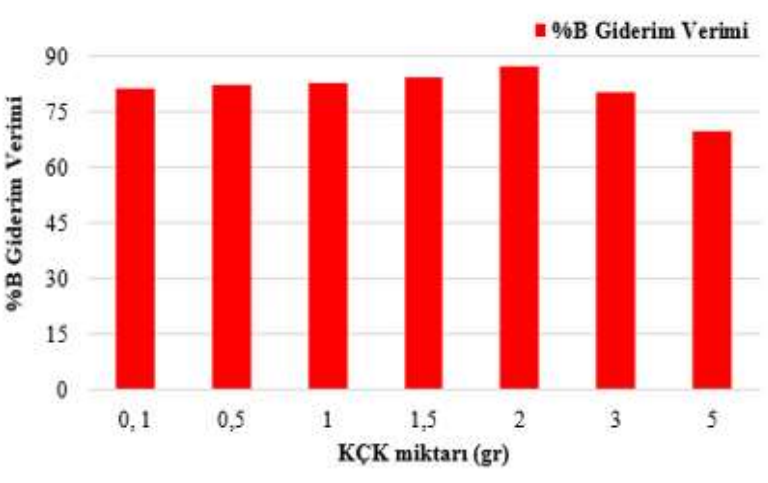

(d) Bor giderimine KÇ dozunun etkisi

Şekil 5. Bor gideriminin farklı adsorpsiyon parametrelerine göre değişimleri 


\subsection{KÇK Temas Süresi}

Temas süresi adsorpsiyon prosesinin dengeye ulaşabilmesi için gereken süre olup bor ile yapılan adsorpsiyon çalışmaları incelendiği zaman adsorpsiyon dengesi genellikle uzun zaman dilimlerinde meydana gelebilmektedir. Şekil 5c'de görülebileceği gibi, KÇK ve bor arasındaki temas süresi ilişkisi anlamlı bir etkiye sahiptir. Borun adsorpsiyonu, KÇK üzerinde 5-120 dakika aralığında zamanın bir fonksiyonu olarak incelenmiştir. Verimlilik başlangıçta hızlı bir şekilde artmış ve KÇK için 5 dakikalık temas süresinde maksimum \%87.45 düzeyine ulaşmıştır. Adsorpsiyon işleminin birinci evresindeki daha yüksek giderme oranı, bor adsorpsiyonu için KÇK'nın daha büyük boş yüzey alanı nedeniyle olabilir. Önceki literatürdeki birçok çalışmanın bulguları çalışmanın sonuçları ile uyumludur [46-49].

\subsection{Kesikli Deneylerde Uygulanan İzoterm ve Kinetik Modeller}

Maksimum adsorpsiyon kapasitesini değerlendirmek ve adsorpsiyon mekanizmasını belirlemek için, deneysel verileri tanımlayan Langmuir (L), Freundlich (F), Dubinin-Radushkevich (D-R) ve Temkin (T) izoterm modelleri kullanılmıştır. Adsorpsiyon dengelerini tanımlamak için kullanılan en gelişmiş denklemler esas olarak Langmuir ve Freundlich'tir. Elde edilen izoterm parametrelerine ait katsayılar, korelasyon faktörleri $\left(\mathrm{R}^{2}\right)$ ve matematiksel denklemeler Tablo 2'de listelenmiştir [50-55]. Ayrıca, bir Langmuir izoterminin temel özellikleri, boyutsuz sabit ayırma faktörü olan $\mathrm{R}_{\mathrm{L}}$ ile ifade edilebilir. $\mathrm{R}_{\mathrm{L}}$ parametresi izotermin şeklini aşağıdaki şekilde gösterir: $\mathrm{R}_{\mathrm{L}}>1$, elverişsiz; $\mathrm{R}_{\mathrm{L}}=1$, doğrusal; $0<\mathrm{R}_{\mathrm{L}}<1$, uygun ve $\mathrm{R}_{\mathrm{L}}=0$, geri döndürülemez. Hesaplanan maksimum adsorpsiyon kapasitesi qm, $20^{\circ} \mathrm{C}^{\prime} \mathrm{de}, 5$ dakika temas süresi ve $282.1 \mathrm{mg} / \mathrm{L}$ 'lik başlangıç bor konsantrasyonu ile $12.61 \mathrm{mg} / \mathrm{g}$ olarak belirlenmiştir.

Şekil 6 ve 7, KÇK üzerindeki borun adsorpsiyon izotermlerini ve Langmuir, Freundlich, D-R ve Temkin adsorpsiyon modellerinin deneysel verilere uygunluğunu göstermektedir. Tablo 3'de görülebileceği gibi, Langmuir modeli, en yüksek korelasyon katsayısı nedeniyle (0.999) KÇK üzerindeki bor adsorpsiyonu deneysel verisine en iyi sonucu verdi. Boyutsuz sabit ayırma faktörü, $\mathrm{R}_{\mathrm{L}}$, $282.1 \mathrm{mg} / \mathrm{L}$ başlangıç bor konsantrasyonu için 0.489 olup $0<\mathrm{R}_{\mathrm{L}}<1$ aralı̆̆ında Langmuir için uygun adsorpsiyon göstermiştir. Ancak diğer modellerde kötü bir uyumsuzluk söz konusudur. Bu sonuçlar önceki çalışmaların sonuçları ile uygun düzeydedir [56-58].

Tablo 2. Isotherm models, their linear forms and respective coefficient

\begin{tabular}{|c|c|c|c|}
\hline Modeller & Denklemler & Katsa & \\
\hline \multirow{4}{*}{ Langmuir } & $\mathrm{q}_{\mathrm{m}} \mathrm{K}_{\mathrm{L}} \mathrm{C}_{\mathrm{e}}$ & $\mathrm{q}_{\mathrm{m}}(\mathrm{mg} / \mathrm{g})$ & 12.61 \\
\hline & $\mathrm{q}_{\mathrm{e}}=\overline{1+\mathrm{K}_{\mathrm{L}} \mathrm{C}_{\mathrm{e}}}$ & $\mathrm{K}_{\mathrm{L}}(\mathrm{L} / \mathrm{mg})$ & 0.064 \\
\hline & & $\mathrm{R}_{\mathrm{L}}$ & 0.489 \\
\hline & $\mathrm{K}_{\mathrm{L}}=\overline{1+\mathrm{K}_{\mathrm{L}} \mathrm{xC}_{\mathrm{e}}}$ & $\mathrm{R}^{2}$ & 0.999 \\
\hline \multirow{3}{*}{ Freundlich } & \multirow{3}{*}{$\mathrm{q}_{\mathrm{e}}=\mathrm{K}_{\mathrm{F}} \sqrt[\mathrm{n}]{\mathrm{C}_{\mathrm{e}}}$} & $\mathrm{K}_{\mathrm{F}}(\mathrm{L} / \mathrm{mg})$ & 9.710 \\
\hline & & $\mathrm{n}$ & 20.12 \\
\hline & & $\mathrm{R}^{2}$ & 0.479 \\
\hline \multirow{2}{*}{ Tempkin } & \multirow{2}{*}{$\mathrm{q}_{\mathrm{e}}=\mathrm{q}_{\mathrm{m}} \ln \left(\mathrm{K}_{\mathrm{T}} \mathrm{C}_{\mathrm{e}}\right)$} & $\mathrm{K}_{\mathrm{T}}$ & 346.94 \\
\hline & & $\mathrm{R}^{2}$ & 0.561 \\
\hline \multirow{3}{*}{ D-R } & \multirow{3}{*}{$\operatorname{Inq} q_{e}=\ln q_{\max }-\beta \varepsilon^{2}$} & $\beta\left(\mathrm{mol}^{2} / \mathrm{j}^{2}\right)$ & 0.241 \\
\hline & & $\mathrm{E}(\mathrm{kj} / \mathrm{mol})$ & 1.440 \\
\hline & & $\mathrm{R}^{2}$ & 0.433 \\
\hline \multirow{3}{*}{ Yalancı Birinci derece } & \multirow{3}{*}{$\operatorname{In}\left(\mathrm{q}_{\mathrm{e}}-\mathrm{q}_{\mathrm{t}}\right)=\operatorname{In} \mathrm{q}_{\mathrm{e}}-\mathrm{k}_{1} \times \mathrm{t}$} & $\mathrm{k}_{1}(1 / \min )$ & 0.018 \\
\hline & & $\mathrm{q}_{\mathrm{e}}(\mathrm{mg} / \mathrm{g})$ & 2.127 \\
\hline & & $\mathrm{R}^{2}$ & 0.196 \\
\hline \multirow{3}{*}{ Yalancı İkinci Derece } & \multirow{3}{*}{$\frac{\mathrm{t}}{\mathrm{q}_{\mathrm{t}}}=\frac{1}{\mathrm{k}_{2} \times \mathrm{q}_{\mathrm{e}}^{2}}+\frac{1}{\mathrm{q}_{\mathrm{w}}} \mathrm{t}$} & $\mathrm{k}_{2}(\mathrm{~g} / \mathrm{mg} / \mathrm{min})$ & 0.030 \\
\hline & & $\mathrm{q}_{\mathrm{e}}(\mathrm{mg} / \mathrm{g})$ & 12.193 \\
\hline & & $\mathrm{R}^{2}$ & 0.987 \\
\hline \multirow{2}{*}{ Intrapartikül difüzyon } & \multirow{2}{*}{$\mathrm{q}_{\mathrm{t}}=\mathrm{k}_{\mathrm{d}} \times \mathrm{t}^{0.5}$} & $\mathrm{k}_{\mathrm{d}}$ & 0.409 \\
\hline & & $\mathrm{R}^{2}$ & 0.657 \\
\hline \multirow{3}{*}{ Evolvich } & \multirow{3}{*}{$\mathrm{q}_{\mathrm{t}}=\frac{1}{\beta} \operatorname{In} \alpha \beta+\frac{1}{\beta} \operatorname{Int}$} & $\alpha$ & 2.984 \\
\hline & & $\beta$ & 0.255 \\
\hline & & $\mathrm{R}^{2}$ & 0.156 \\
\hline
\end{tabular}

KL: Langmuir, KF: Freundlich, qm: Maksimum adsorpsiyon kapasitesi, KT: Tempkin; k1: Birinci derece kinetic, k2: İkinci derece kinetic 


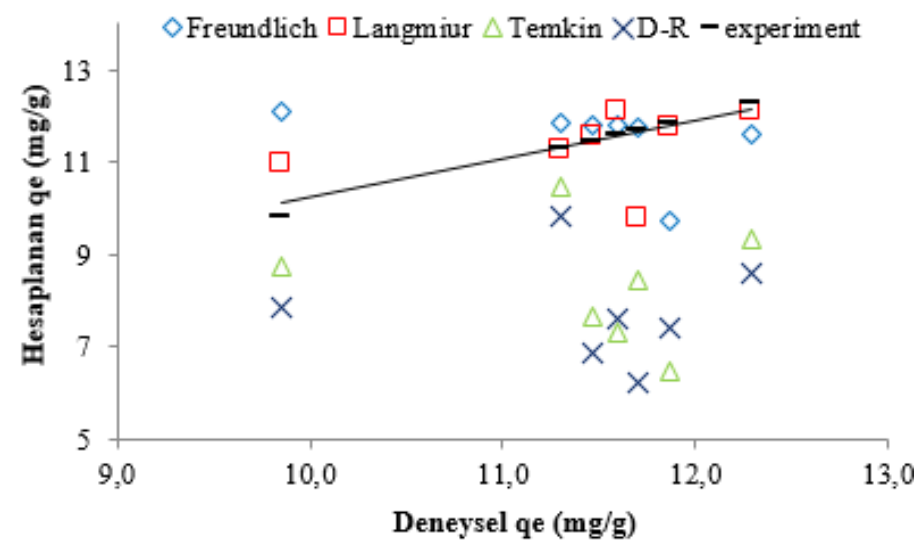

Şekil 6. KÇK ile bor adsorpsiyonunun deneysel izoterm eğrileri
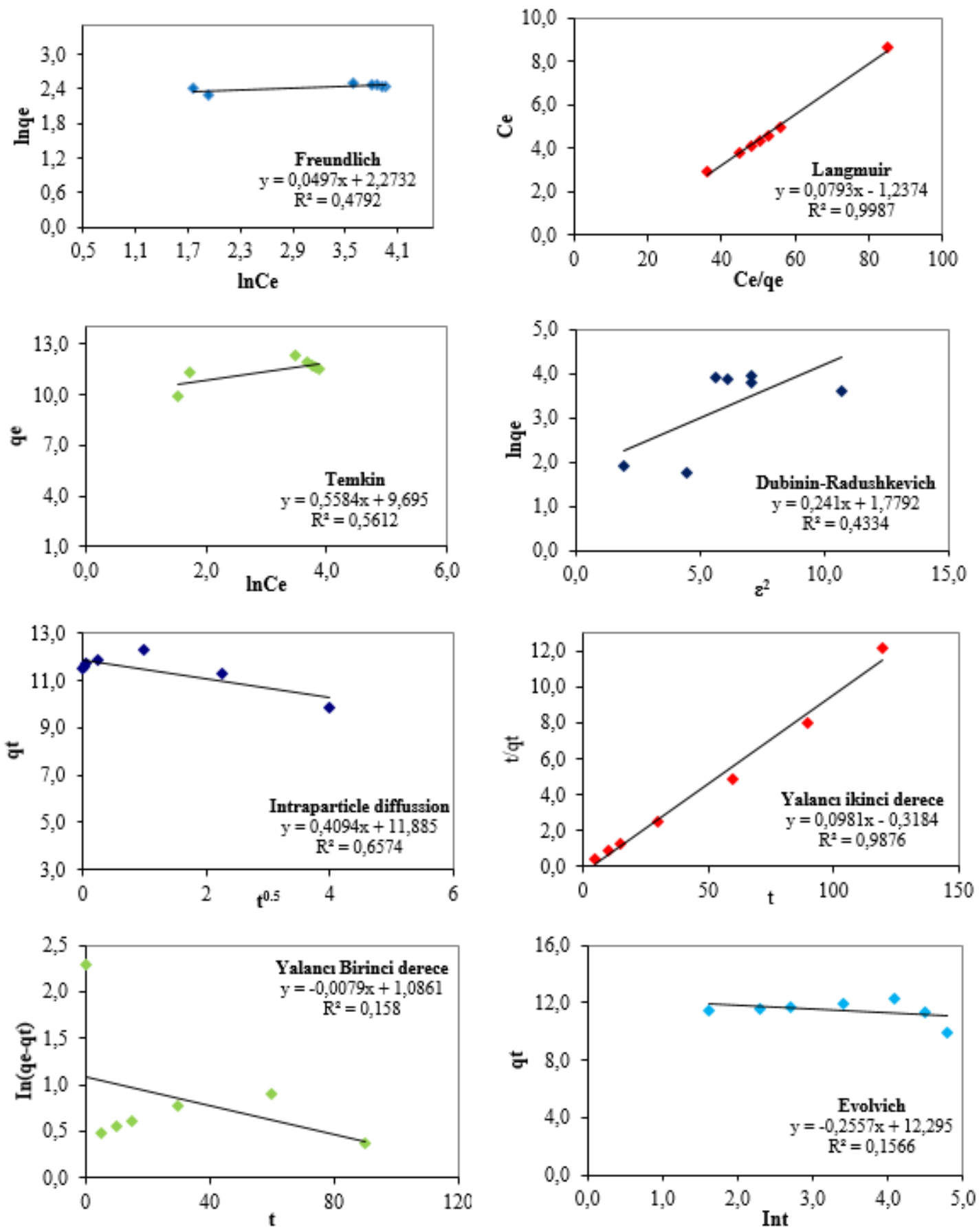

Şekil 7. KÇK ile bor adsorpsiyonunun izoterm ve kinetik eğrileri 
Katı-faz ara kesitinde KÇK üzerine borun giderimini belirlemek için adsorpsiyon kinetiği çalışması önemlidir. Bu çalışmada adsorpsiyon verilerini tanımlamak ve KÇK'nın etkinliğini belirlemek için yaygın olarak kullanılan yalancı birinci dereceden, yalancı ikinci dereceden kinetik (YIDK), intrapartikül difüzyon ve Evolvich dahil olmak üzere çeşitli kinetik modeller test edilmiştir (Tablo 2). Adsorpsiyon kapasiteleri ve modellerden hesaplanan değerler Tablo 2'de verilmiştir. Kinetik veri bulguları karşılaştırıldığında, en iyi korelasyon katsayısının sözde ikinci dereceden model ile elde edildiği söylenebilir. Ayrıca, sözde ikinci dereceden modelden elde edilen değerler deneysel olarak KÇK adsorpsiyonunun başarılı sonuçlandığını göstermektedir. Borun farklı adsorbentler tarafından adsorpsiyonuna odaklanan önceki çalışmalara göre, borun giderilmesi sözde ikinci dereceden kinetik modelleme için uygunluk göstermektedir $[59,60]$.

\subsection{Literatürde Rapor Edilen Farklı Adsorbentlerle Bor Gideriminin Karşılaştırılması}

Borun sulu çözeltilerden adsorpsiyonu için farklı adsorbentlerin adsorpsiyon yöntemi ile giderimini gösteren liste Tablo 3'te verilmiştir. Görüldüğü gibi, bor için KÇK'nın gözlemlenen arıtım verimleri diğer adsorbentlerle karşılaştırılabilir nitelikte olup, bu durum KÇK'nın bor çalışmalarında alternatif bir adsorbent olabileceğini net bir şekilde göstermektedir.

Table 3. Düşük maliyetli bazı adsorbentlerin bor adsorpsiyon kriterlerinin karşılaştırılması

\begin{tabular}{|c|c|c|c|c|c|}
\hline Adsorbent & Optimum Koşullar & $\%$ Verim & İzoterm & Kinetik & Kaynaklar \\
\hline Uçucu kül & pH: 10 , doz: $100 \mathrm{~g}, \mathrm{t}: 25$ saat & 94 & $\mathrm{~L} / \mathrm{F}$ & YİDK & {$[61]$} \\
\hline Al-Arıtım atıkları & $\mathrm{pH}: 8.5$, doz: $25 \mathrm{~g}$ & 24.5 & $\mathrm{~L}$ & YİDK & [62] \\
\hline Hurma Külü & pH: 7, doz: $6 \mathrm{~g}, \mathrm{t}: 12$ saat & 65.7 & - & YİDK & [63] \\
\hline Bentonit/Zeolit & pH: 9 , doz: $50 \mathrm{~g}, \mathrm{t}: 2$ saat & 43 & $\mathrm{~L} / \mathrm{F}$ & - & {$[64]$} \\
\hline Ceviz-Pirinç kabuğu & pH: 7, doz: 2 g, t: 48 saat & 82 & $\mathrm{~L}$ & - & {$[64]$} \\
\hline Bentonit-Magnetit & pH: 2-12, doz: 1 g, t: 0.5 saat & 95 & $\mathrm{~L} / \mathrm{F}$ & YİDK & [65] \\
\hline Kitosan & pH: 8 , doz: $0.15 \mathrm{~g}$, t: 0.5 saat & 70 & $\mathrm{~L} / \mathrm{F}$ & YİDK & {$[66]$} \\
\hline Sepiyolit & $\mathrm{pH}: 9$, doz: $0.5 \mathrm{~g}, \mathrm{t}: 30$ dakika & 43 & - & - & [9] \\
\hline Lantanyum-Kitosan & $\mathrm{pH}: 9.8$, doz: $5.34 \mathrm{~g}, \mathrm{t}: 24$ saat & 58 & $\mathrm{~L} / \mathrm{F}$ & YİDK & {$[58]$} \\
\hline Sezyum-Kitosan & pH: 7, doz: 3 g, t: 24 saat & 62 & $\mathrm{~L} / \mathrm{F}$ & YİDK & {$[45]$} \\
\hline ZIF67 nanomalzeme & $\mathrm{pH}: 4$, doz: $3 \mathrm{~g}, \mathrm{t}: 24$ saat & 94.1 & $\mathrm{~L}$ & - & [56] \\
\hline Zirkonyum-Kitosan & pH: 8 , doz: 2 g, t: 24 saat & 54.3 & $\mathrm{~L} / \mathrm{F}$ & YİDK & [31] \\
\hline KÇK & $\mathrm{pH}: 5.13$, doz: $2 \mathrm{~g}, \mathrm{t}: 5$ dakika & 92 & $\mathrm{~L}$ & YİDK & Bu çalışma \\
\hline
\end{tabular}

\section{Sonuç ve Öneriler}

Mevcut deneysel çalışma sonuçları, bor elementinin KÇK üzerine adsorpsiyonunun $\mathrm{pH}$, temas süresi ve KÇK miktarına bağlı olduğunu göstermiştir. KÇK'nın maksimum arıtma verimi, ideal şartlarda bor için yaklaşık \% 88 olarak hesaplanmıştır. KÇK üzerine bor adsorpsiyon işlemi için optimum koşullar KÇK dozu $=2 \mathrm{~g} / \mathrm{L}, \mathrm{pH}=5.13$ ve temas süresi $=5$ dakika olarak elde edilmiştir. Langmuir izotermine dayalı maksimum adsorpsiyon kapasitesi ve korelasyon katsayıs $\left(\mathrm{R}^{2}\right) 12.61 \mathrm{mg} / \mathrm{g}$ ve 0.999 şeklinde olup borun KÇK üzerine adsorpsiyonu yalancı ikinci derece kinetiği ile iyi tanımlanmıştır. Boru sulu çözeltilerden giderebilmek için, bu çalışma açıkça KÇK'nın pratik, etkili ve düşük maliyetli, yüksek kapasiteli, çevre dostu bir adsorbent olarak kullanılmasının etkinliğini göstermektedir.

\section{Yazarların Katkısı}

Çalışmada tüm katkı yazara aittir.

\section{Çıkar Çatışması Beyanı}

Yazarlar arasında herhangi bir çıkar çatışması bulunmamaktadır.

\section{Araştırma ve Yayın Etiği Beyanı}

Yapılan çalışmada araştırma ve yayın etiğine uyulmuştur. 


\section{Kaynaklar}

[1] Türker O.C., Yakar A., Türe C., Saz Ç. 2019. Cost-Effectiveness of Boron (B) Removal from Irrigation Water: An Economic Water Treatment Model (EWTM) for Farmers to Prevent Boron Toxicity. Environmental Science and Pollution Research, 26: 18777-18789.

[2] Güven E.D., Güler E., Akıncı G., Bölükbaş A. 2017. Influencing Factors in the Removal of High Concentrations of Boron by Electrocoagulation. Journal of Hazardous, Toxic, and Radioactive Waste, 22 (2): 04017031.

[3] Simcox L., A. Pereira R.P., Wellington E.M., Macpherson J.V. 2019. Boron Doped Diamond as a Low Biofouling Material in Aquatic Environments: Assessment of Pseudomonas Aeruginosa Biofilm Formation. ACS Applied Materials \& Interfaces, 11: 25024-25033.

[4] Helvacı C. 2015. Bor Yataklarının Mineral ve Kimyası Yönünden Genel Değerlendirilmesi ve Gelecek Öngörüsü. Madencilik ve Yerbilimleri Dergisi, 6 (47): 66-78.

[5] Tagliabue M., Reverber A.P., Bagatin R. 2014. Boron Removal from Water: Needs, Challenges and Perspectives, Journal of Cleaner Production, 77: 56-64.

[6] Sönmez N.A. 2014. Sulardan Adsorpsiyon Yöntemi ile Bor Giderimi. Yüksek Lisans Tezi, Pamukkale Üniversitesi, Fen Bilimleri Enstitüsü, Denizli.

[7] Kabay N., Bryjak M., Hilal N. 2015. Boron separation processes. Elsevier Publisher, Amsterdam.

[8] Tombal T.D., Özkan Ş.G., Ünver İ.K., Osmanlıŏlu A.E. 2016. Bor Bileşiklerinin Özellikleri, Üretimi, Kullanımı ve Nükleer Reaktör Teknolojisinde Önemi. Journal of Boron, 1 (2): 86-95.

[9] Arıkan T. 2019. Sulu Çözeltideki Bor İyonlarının Sepiyolit ile Giderimi. KSÜ Tarım ve Doğa Dergisi, 22 (2): 333-338.

[10] Başkan M.B., Atalay N. 2014. İçme ve Sulama Sularında Bor Kirliliği ve Bor Giderme Yöntemleri. Pamukkale Üniversitesi Mühendislik Bilimleri Dergisi, 20 (3): 78-84.

[11] WHO. 2011. http://whqlibdoc.who.int/publications/2011/9789241548151_eng.pdf. (Erişim Tarihi: 29.12.2019).

[12] EU. 1998. https://eur-lex.europa.eu/legal-content/EN/TXT/?uri=CELEX\%3A31998L0083 (Erişim Tarihi: 29.12.2019).

[13] Jung J., Choi H., Hong S., Yoon S.J., Kim T.H., Lee J.Y., Hong Y.T., So S. 2020. SurfaceInitiated ATRP of Glycidyl Methacrylate in the Presence of Divinylbenzene on Porous Polystyrene-Based Resins for Boron Adsorption. Desalination, 473: 114166.

[14] Weidner E., Ciesielczyk F. 2019. Removal of Hazardous Oxyanions from the Environment Using Metal-Oxide-Based Materials. Materials, 12 (6): 927.

[15] Tulun Ş., Bahadır T., Şimşek İ., Karataş M. 2019. The Removal of Nickel Ions with Walnut Shell. Turkish Journal of Engineering, 3 (2): 102-105.

[16] Meng F., Ma W., Wu L., Hao H., Xin L., Chen Z., Wang M. 2018. Selective and Efficient Adsorption of Boron (III) from Water by 3D Porous CQDs/LDHs with Oxygen-Rich Functional Groups. Journal of the Taiwan Institute of Chemical Engineers, 83: 192-203.

[17] Türker O.C., Baran T. 2017. Evaluation and Application of an Innovative Method Based on Various Chitosan Composites and Lemna gibba for Boron Removal from Drinking Water. Carbohydrate Polymers, 166: 209-218.

[18] Wolska J., Bryjak M. 2013. Methods for Boron Removal from Aqueous Solution-a Review. Desalination, 310: 18-24.

[19] Türker O.C., Baran T. 2018. A Combination Method Based on Chitosan Adsorption and Duckweed (Lemna gibba L.) Phytoremediation for Boron (B) Removal from Drinking Water. International journal of phytoremediation, 20 (2): 175-183.

[20] Deniz F., Akarsu C. 2018. Operating Cost and Treatment of Boron from Aqueous Solutions by Electrocoagulation in Low Concentration. Global Challenges, 2 (5-6): 1800011.

[21] Guan Z., Lv J., Bai P., Guo X. 2016. Boron Removal from Aqueous Solutions by Adsorption-a Review. Desalination, 383: 29-37.

[22] Al Haddabi M., Ahmed M., Al. Jebri Z., Vuthaluru H., Znad H., Al. Kindi M. 2016. Boron Removal from Seawater Using Date Palm (Phoenix dactylifera) Seed Ash. Desalination and Water Treatment, 57 (11): 5130-5137.

[23] Xi Z., Qilong R., Yiwen Y., Huabin X., Baogen S., Zongbi B. 2015. Materials for Boron Adsorption. Progress in Chemistry, 27 (1): 125-134. 
[24] Shireen F., Nawaz M.A., Chen C., Zhang Q., Zheng Z., Sohail H., Sun J., Cao H., Huang Y., Bie Z. 2018. Boron: Functions and Approaches to Enhance Its Availability in Plants for Sustainable Agriculture. International Journal of Molecular Sciences, 19 (7): 1856.

[25] Laçin B., Ertit Taştan B., Dönmez G. 2015. Detection of Boron Removal Capacities of Different Microorganisms in Wastewater and Effective Removal Process. Water Science and Technology, 72 (10): 1832-1839.

[26] Çelebi H. 2019. The Applicability of Evaluable Wastes for the Adsorption of Reactive Black 5. International Journal of Environmental Science and Technology, 16 (1): 135-146.

[27] Roongruangsri W., Bronlund J.E. 2016. Effect of Air-Drying Temperature on Physico-Chemical, Powder Properties and Sorption Characteristics of Pumpkin Powders. International Food Research Journal, 23: 962-972.

[28] Njoku V.O., Foo K.Y., Hameed B.H. 2013. Microwave-Assisted Preparation of Pumpkin Seed Hull Activated Carbon and Its Application for the Adsorptive Removal of 2, 4Dichlorophenoxyacetic Acid. Chemical Engineering Journal, 215: 383-388.

[29] Kluczka J., Pudlo W., Krukiewicz K. 2019. Boron Adsorption Removal by Commercial and Modified Activated Carbons. Chemical Engineering Research and Design, 147: 30-42.

[30] Sun L., Huang J., Liu H., Zhang Y., Ye X., Zhang H., Wu A., Wu Z. 2018. Adsorption of Boron byCA@KH-550@EPH@ NMDG (CKEN) with Biomass Carbonaceous Aerogels as Substrate. Journal of Hazardous Materials, 358: 10-19.

[31] Kluczka J., Gnus M., Kazek-Kęsik A., Dudek G. 2018. Zirconium-Chitosan Hydrogel Beads for Removal of Boron from Aqueous Solutions. Polymer, 150: 109-118.

[32] Sarri S., Misaelides P., Zamboulis D., Warchoł J. 2018. Boron Removal from Aqueous Solutions by a Polyethylenimine-Epichlorohydrin Resin. Journal of the Serbian Chemical Society, 83 (2): 251-264.

[33] Chen T., Wang Q., Lyu J., Bai P., Guo X. 2020. Boron Removal and Reclamation by Magnetic Magnetite (Fe3O4) Nanoparticle: An Adsorption and Isotopic Separation Study. Separation and Purification Technology, 231: 115930.

[34] Sanfeliu C., Martínez-Máñez R., Sancenón F., Soto J., Amorós P., Azaïs T., Marcos M.D. 2018. 11B-MAS NMR Approach to the Boron Adsorption Mechanism on a Glucose-Functionalised Mesoporous Silica Matrix. Microporous and Mesoporous Materials, 266: 232-241.

[35] Jaouadi M., Hbaieb S., Guedidi H., Reinert L., Amdouni N., Duclaux L. 2017. Preparation and Characterization of Carbons from $\beta$-cyclodextrin Dehydration and from Olive Pomace Activation and Their Application for Boron Adsorption. Journal of Saudi Chemical Society, 21 (7): 822-829.

[36] Kluczka J., Dudek G., Kazek-Kęsik A., Gnus M. 2019. Chitosan Hydrogel Beads Supported with Ceria for Boron Removal. International Journal of Molecular Sciences, 20 (7): 1567.

[37] Tlili A., Dridi I., Attaya R., Gueddari M. 2019. Boron Characterization, Distribution in ParticleSize Fractions, and Its Adsorption-Desorption Process in a Semiarid Tunisian Soil. Journal of Chemistry, 2019: 1-8.

[38] Nozawa S., Sato T., Otake T. 2018. Effect of Dissolved Silica on Immobilization of Boron by Magnesium Oxide. Minerals, 8 (2): 76.

[39] Montalvo Andia J.P., Yokoyama L., Teixeir, C., Alberto L. 2018. Study of the Equilibrium, Kinetics, and Thermodynamics of Boron Removal from Waters with Commercial Magnesium Oxide. International Journal of Chemical Engineering, 2018: 1-10.

[40] Bodzek M. 2016. The Removal of Boron from the Aquatic Environment-State of the Art, Desalination and Water Treatment, 57 (3): 1107-1131.

[41] Kluczka J., Trojanowska J., Zołotajkin M. 2015. Utilization of Fly Ash Zeolite for Boron Removal from Aqueous Solution. Desalination and Water Treatment, 54 (7): 1839-1849.

[42] Affam A.C., Wong C.C., Seyam M.A., Matt C.A.A.F., Sumbai J.L.A., Evut, A.M. 2018. Preparation, Characterization and Adsorption Study of Granular Activated Carbon/Iron Oxide Composite for the Removal of Boron and Organics from Wastewater. E3S Web of Conferences 34: 02006.

[43] Tang Y.P., Luo L., Thong Z., Chung T.S. 2017. Recent Advances in Membrane Materials and Technologies for Boron Removal. Journal of Membrane Science, 541: 434-446. 
[44] Bertagnolli C., Grishin A., Vincent T., Guibal E. 2017. Boron Removal by a Composite Sorbent: Polyethylenimine/Tannic Acid Derivative Immobilized in Alginate Hydrogel Beads. Journal of Environmental Science and Health, Part A, 52 (4): 359-367.

[45] Kluczka J., Gnus M., Dudek G., Turczyn R. 2017. Removal of Boron from Aqueous Solution by Composite Chitosan Beads, Separation Science and Technology, 52 (9): 1559-1571.

[46] Abdelnour S.A., El-Hack M.E.A., Swelum A.A., Perillo A., Losacco C. 2018. The Vital Roles of Boron in Animal Health and Production: A Comprehensive Review. Journal of Trace Elements in Medicine and Biology, 50: 296-304.

[47] Demirçivi P., Saygıll G.N. 2018. Comparative Study of Modified Expanded Perlite with Hexadecyltrimethylammonium-Bromide and Gallic Acid for Boron Adsorption. Journal of Molecular Liquids, 254: 383-390.

[48] Al-Ghouti M.A., Salih N.R. 2018. Application of Eggshell Wastes for Boron Remediation from Water. Journal of Molecular Liquids, 256: 599-610.

[49] Wang J., Zhuang S. 2017. Removal of Various Pollutants from Water and Wastewater by Modified Chitosan Adsorbents. Critical Reviews in Environmental Science and Technology, 47 (23): 2331-2386.

[50] Ho Y.S., McKay G. 1999. Pseudo-Second Order Model for Sorption Processes. Process biochemistry, 34 (5): 451-465.

[51] Hall K.R., Eagleton L.C., Acrivos A., Vermeulen T. 1966. Pore-and Solid-Diffusion Kinetics in Fixed-Bed Adsorption Under Constant-Pattern Conditions. Industrial \& Engineering Chemistry Fundamentals, 5 (2): 212-223.

[52] Dubinin M.M., Radushkevich L.V. 1947. Equation of the Characteristic Curve of Activated Charcoal, Proceedings of the Academy of Sciences. Physical Chemistry Section, 55: 331-337.

[53] Temkin M., Pyzhev V. 1940. Recent Modifications to Langmuir Isotherms. Acta Physical Chemistry, 12: 217-225.

[54] Langmuir I. 1916. The Constitution and Fundamental Properties of Solids and Liquids Part I. Solids. Journal of the American Chemical Society, 38 (11): 2221-2295.

[55] Freundlich H.M.F. 1906. Uber Die Adsorption in Lasungen. Journal of Physical Chemistry, 57: 370-385.

[56] Zhang J., Cai Y., Liu, K. 2019. Extremely Effective Boron Removal from Water by Stable Metal Organic Framework ZIF-67. Industrial \& Engineering Chemistry Research, 58 (10): 4199-4207.

[57] Babiker E., Al-Ghouti M.A., Zouari N., McKay G. 2019. Removal of Boron from Water Using Adsorbents Derived from Waste Tire Rubber. Journal of Environmental Chemical Engineering, 7 (2): 102948.

[58] Kluczka J., Dudek G., Kazek-Kęsik A., Gnus M., Krzywiecki M., Mitko K., Krukiewicz K. 2019. The Use of Lanthanum Ions and Chitosan for Boron Elimination from Aqueous Solutions. Polymers, 11 (4): 718.

[59] Duran H., Yavuz E., Sismanoglu T., Senkal B.F. 2019. Functionalization of Gum Arabic Including Glycoprotein and Polysaccharides for the Removal of Boron. Carbohydrate polymers, 225: 115139.

[60] Demey H., Barron-Zambrano J., Mhadhbi T., Miloudi H., Yang Z., Ruiz M., Sastre A.M. 2019. Boron Removal from Aqueous Solutions by Using a Novel Alginate-Based Sorbent: Comparison with $\mathrm{Al}_{2} \mathrm{O}_{3}$ Particles. Polymers, 11 (9): 1509.

[61] Yüksel S., Yürüm Y. 2009. Removal of Boron from Aqueous Solutions by Adsorption Using Fly Ash, Zeolite, and Demineralized Lignite. Separation Science and Technology, 45 (1): 105-115.

[62] Irawan C., Liu J.C., Wu C.C. 2011. Removal of Boron Using Aluminum-Based Water Treatment Residuals (Al-WTRs). Desalination, 276 (1-3): 322-327.

[63] Chieng H.J., Chong M.F. 2013. Boron Adsorption on Palm Oil Mill Boiler (POMB) Ash Impregnated with Chemical Compounds. Industrial \& Engineering Chemistry Research, 52 (41): 14658-14670.

[64] Jalali M., Rajabi F., Ranjbar, F. 2016. The Removal of Boron from Aqueous Solutions Using Natural and Chemically Modified Sorbents. Desalination and Water Treatment, 57 (18): 82788288 . 
[65] Masindi V., Gitari M.W., Tutu H., Debeer M. 2016. Removal of Boron from Aqueous Solution Using Magnesite and Bentonite Clay Composite. Desalination and Water Treatment, 57 (19): 8754-8764.

[66] Bursalı E.A., Seki Y., Seyhan S., Delener M., Yurdakoç M. 2011. Synthesis of Chitosan Beads as Boron Sorbents. Journal of Applied Polymer Science, 122 (1): 657-665. 